\title{
MONITORING OF 14 MeV NEUTRONS
}

\author{
G. Ban ${ }^{1}$, A. Billebaud ${ }^{2}$, R. Brissot ${ }^{2}$, J.M. Fontbonne ${ }^{1}$, C. Le Brun ${ }^{2}$, \\ F.R. Lecolley ${ }^{1}$, J.F. Lecolley ${ }^{1}$, J.L. Lecouey ${ }^{1}$, E. Liatard ${ }^{2}$, N. Marie ${ }^{1}$ \\ and J.C. Steckmeyer ${ }^{1 *}$ \\ 1 Laboratoire de Physique Corpusculaire, IN2P3/CNRS, ENSICAEN, UCBN, \\ 14050 Caen Cedex 04, France \\ 2 Laboratoire de Physique Subatomique et de Cosmologie, IN2P3/CNRS, UJF, INPG, \\ 38026 Grenoble Cedex, France \\ E-mail: Jean-Claude.Steckmeyer@lpccaen.in2p3.fr
}

\begin{abstract}
Long-lived fission products and minor actinides produced in nuclear power plants are the most radiotoxic nuclear wastes. They can be transmuted into stable nuclei or into nuclei with shorter lifetime thanks to the so-called Accelerator Driven Systems (ADS), consisting of the coupling of an intense high energy proton beam, a spallation target and a sub-critical reactor core. For safety reasons, an on-line and robust measurement of the reactivity during loading and power operation is mandatory. The investigation of the relationship between the current of the accelerator and the power level (or neutron flux) of the reactor appears to be powerful, any change in reactivity being accessible through the measurement of the current and the flux. Such a relationship will be studied in an experiment to be performed at the YALINA facility (JIPNR Sosny - Belarus) in the framework of the EUROTRANS IP ( $\left.6^{\text {th }} \mathrm{FP}\right)$. At this installation, $14 \mathrm{MeV}$ neutrons are produced in $\mathrm{T}(\mathrm{d}, \mathrm{n})^{4} \mathrm{He}$ reactions by a deuteron beam impinging on a TiT target. Due to the tritium consumption over time, the intensity of the deuteron beam cannot be used for the monitoring of the neutron beam. The source neutron yield itself has to be accessed. This contribution describes the performance of a three-element silicon telescope dedicated to the detection of recoil protons produced in reactions induced by $14 \mathrm{MeV}$ neutrons.
\end{abstract}

International Workshop on Fast Neutron Detectors

University of Cape Town, South Africa

April 3-6, 2006

\section{${ }^{*}$ Speaker}




\section{Introduction}

Studies devoted to the radioactive waste management plan to incinerate the most radiotoxic of them, i.e. minor actinides and long lived fission products. These radioactive wastes can be transmuted in two ways: either as a new fuel in the next generation power plants or in dedicated facilities like the Accelerator Driven Systems (ADS) which consist of the coupling of an intense high energy proton beam, a spallation target and a sub-critical reactor core. For safety reasons, in the latter case, the core reactivity $\rho$ should be known and checked at any time.

In the framework of the I.P. EUROTRANS $\left(6^{\text {th }} \mathrm{FP}\right)$, an experimental program (DM2 ECATS) is dedicated to improving the knowledge about the dynamic behaviour of an ADS and to validating the simulation procedures. In particular, experimental techniques aiming at monitoring a sub-critical assembly have to be developed. For that purpose, the relationship between the accelerator current and the core power should be investigated. Part of this program is being envisaged at the YALINA facility (JIPNR Sosny - Belarus).

The reactivity $\rho$ of a sub-critical assembly can be expressed as follows:

$$
-\rho=C \phi^{*} \frac{S}{R}
$$

In this equation, $\phi^{*}$ is the importance of the neutron source which accounts for a lower fission probability for spallation neutrons than for fission neutrons, $S$ the number of source neutrons per second and $R$ the counting rate of a detector such as a ${ }^{3} \mathrm{He}$ proportional counter or a fission chamber located in the assembly. $C$ is a constant previously calibrated in a well defined $\rho$ configuration. A monitoring over time of the reactivity $\rho$ requires the knowledge of both $S$ and $R$ quantities throughout the duration of the experiment.

At the YALINA facility [1], a deuteron beam impinges on a TiT target. Due to the consumption of T over time, the beam intensity measurement is of no use for evaluating $S$. Instead, the neutron flux produced in the $\mathrm{T}(\mathrm{d}, \mathrm{n})^{4} \mathrm{He}$ reactions has to be determined. This paper deals with the measurement of $14 \mathrm{MeV}$ neutrons by detecting recoil protons with a three element telescope located at $0^{\circ}$ with respect to the deuteron beam.

\section{Experimental conditions}

\subsection{Setup}

The measurements were performed at the PEREN installation of LPSC in Grenoble [2]. A deuteron accelerator (GENEPI) similar to the one used in the MUSE experiment [3] delivers ${ }^{2} \mathrm{H}^{+}$ions at $140-240 \mathrm{keV}$ of energy. The ion beam impinges on a TiT target located in a high purity lead assembly, usually used as a time slowing down spectrometer of dimensions 1.6 x 1.6 $x 1.6 \mathrm{~m}^{3}$. The target is centered in the plane crosswise the beam axis and off-centered by $20 \mathrm{~cm}$ along the beam axis. The frequency of the pulsed deuteron beam may be varied between $10 \mathrm{~Hz}$ and $5 \mathrm{kHz}$. The width of each pulse is of the order of $1 \mu \mathrm{s}$. 
Monitoring of the neutron beam is ensured by the detection of alpha particles created in the $\mathrm{T}(\mathrm{d}, \mathrm{n}) \alpha$ reaction. For that purpose, a silicon detector, $10 \times 10 \mathrm{~mm}^{2}$ covered by a collimator $\phi=3.2 \mathrm{~mm}$ and a $5 \mu \mathrm{m}$ aluminium foil (to reduce low energy electron background), is installed upstream from the TiT target inside the beam guide. Taking advantage of the isotropic emission in the center of mass of the reaction, the absolute number of neutrons per pulse is deduced from the counting rate of the silicon detector after correction for the geometrical efficiency. Typically $10^{5}$ neutrons are produced per pulse of the deuteron beam.

The neutrons produced in the TiT target can be detected without any interaction in the lead block thanks to a $10 \times 10 \mathrm{~cm}^{2}$ channel opened at zero degree. They interact with a converter made of polyethylene. The protons created by elastic scattering are then detected in a silicon telescope consisting of three elements: $300 \mu \mathrm{m}, 300 \mu \mathrm{m}$ and $4 \mathrm{~mm}$ thick, respectively, with an area of $5 \times 5 \mathrm{~cm}^{2}$. Some measurements were also performed with a $500 \mu \mathrm{m}$ thick silicon detector instead of the $4 \mathrm{~mm}$. The range of $14 \mathrm{MeV}$ protons (maximum recoil energy) is $1.28 \mathrm{~mm}$ in silicon material. The converter and the three detectors were arranged in a compact setup (the average gap between two elements was about $7 \mathrm{~mm}$ ) and placed in a metallic box providing a light shielding. The box was located at different distances downstream from the TiT target (see Fig. 1), outside of the lead block. The lead block is of no use in these measurements.

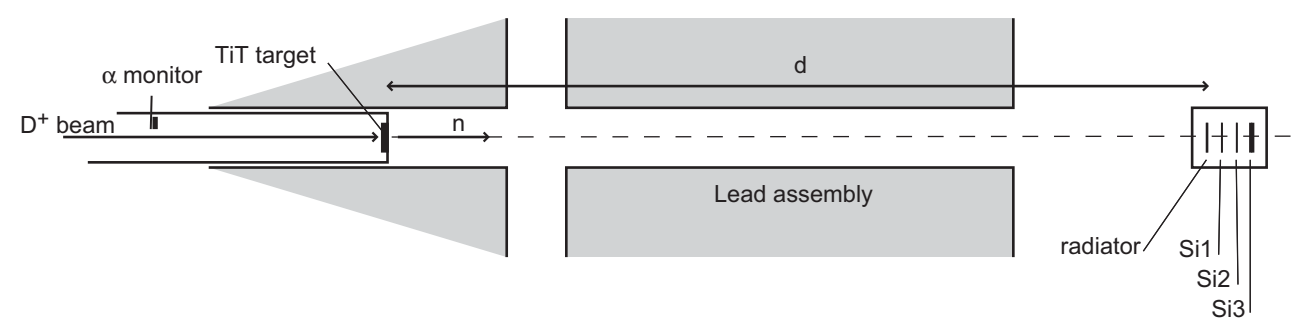

Figure 1: Experiment set-up.

\subsection{Electronics and acquisition}

Each silicon detector signal is split after the preamplifier in a timing filter amplifier and in a spectroscopy amplifier. The fast signals are transformed into NIM logic pulses via constant fraction discriminators, allowing noise rejection and event selection.

The data acquisition is performed by an analog-to-digital converter card developed at the LPSC for a VME crate piloted by a dedicated software on a SUN workstation. This card allows both the energy and time marking of 8 independent inputs. Each analog-digital converter input is triggered by its own logical input. This latter trigs the storage of the time value of a $42 \mathrm{bit}$ time counter fed by a $40 \mathrm{MHz}$ clock and of the 13 bit digitized energy for each event. Data processing, time, energy and coincidence spectra can be performed both on and off-line. A hardware coincidence was also performed with the silicon logical outputs and displayed on a scaler. 


\section{Measurements}

\subsection{Measurements performed as a function of the target-telescope distance}

The box containing the converter and the silicon detectors was placed at different distances $d$ downstream from the target: 109, 170 and $240 \mathrm{~cm}$. For the two extreme positions, measurements were also performed without converter.

In Fig. 2, top row, the energy spectra of each silicon detector are shown when recorded with a single trigger condition (solid lines) and when a triple coincidence is required (dashed area). The coincident spectra are shown again in the bottom row. The requirement of a triple coincidence cleans up the energy spectra. On each detector, an energy threshold of 1-1.5 MeV is clearly seen in the single trigger spectra, due to the tuning of the discriminator units.

The spectra shown in Fig. 2 were recorded when the telescope was located at a distance of $240 \mathrm{~cm}$ from the TiT target. Similar spectra recorded at shorter distances show an increase of high-energy events. These events are due to pile up which is expected to increase when the distance between the TiT target and the telescope decreases.

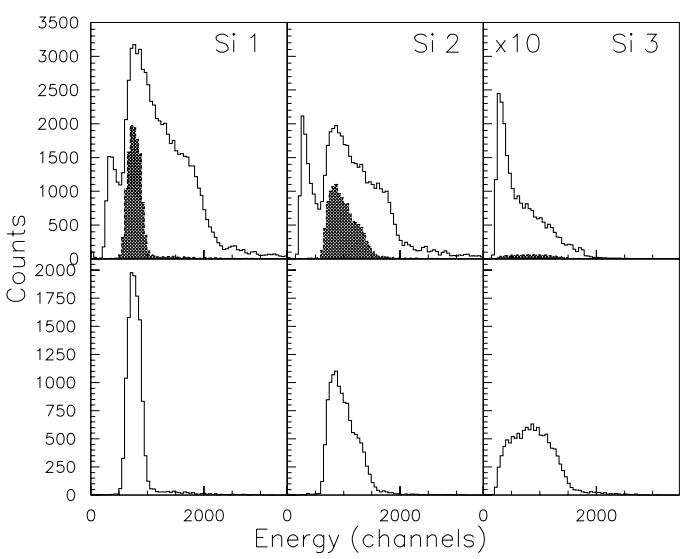

Figure 2: Raw energy spectra in the three silicon detectors: single spectra (solid lines in the top row) and coincident spectra (bottom row and hatched areas in the top row). The number of counts in the upper right histogram has been divided by a factor 10 .

Simulations were performed with the MCNPX code version 2.5. [4]. In Fig. 3, the raw coincident spectra shown in Fig. 2 (bottom row) and the results of the simulations have been superimposed in such a way that their shapes coincide. The implicit calibration performed in this way, on both single and coincident spectra for all measurements, agrees well with a rough energy calibration carried out with a radioactive source emitting alpha particles of $5.5 \mathrm{MeV}$ energy.

In the coincident spectra of silicon 1 and 2, a clear threshold, due to physics and not to electronics or detection, is evidenced at $2 \mathrm{MeV}$. After setting the discriminator thresholds of detectors 1 and 2 at an appropriate level, the counting rate of the logical triple coincidence signal will allow for a relative on-line monitoring of the recoil proton number. As for the threshold of 1-1.5 MeV in the third silicon, it is due to the discriminator threshold. In this detector, the energy spectrum should start at $0 \mathrm{MeV}$ as simulations do. For the sake of comparison, a threshold of $1.5 \mathrm{MeV}$ was introduced in the simulations. 

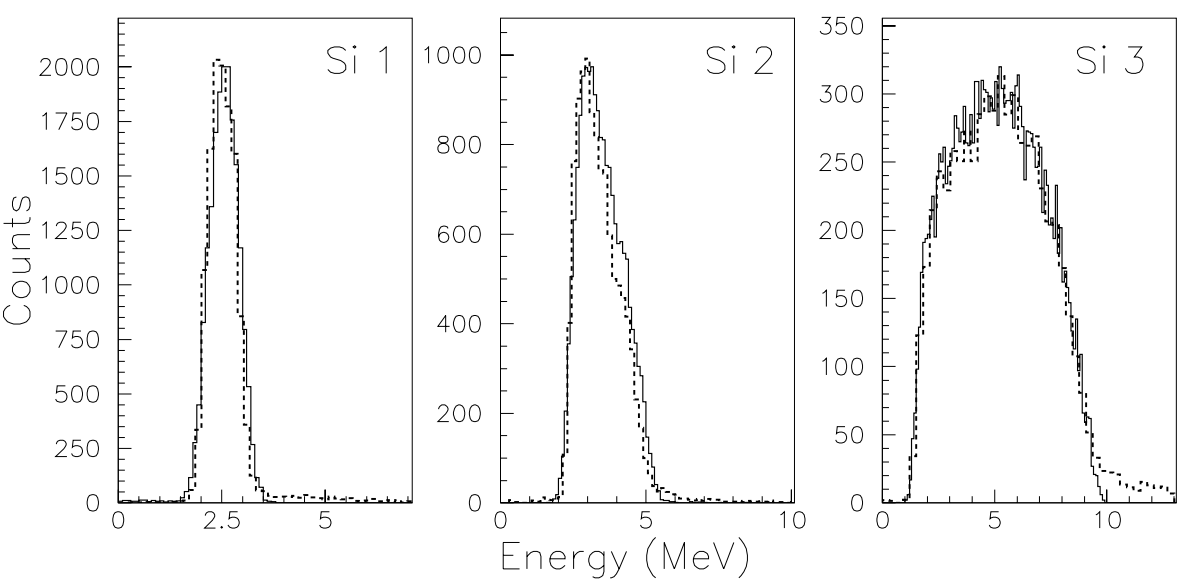

Figure 3: Measured (dashed lines) and simulated (solid lines) energy spectra of recoil protons in the silicon detectors.

In Table 1, the measured number of protons produced per source neutron is given as a function of the distance of the telescope with respect to the TiT target. Also shown in this table are the values obtained with the MCNPX simulations. The discrepancy between measurements and calculations is less than $15 \%$.

\begin{tabular}{|l|l|l|l|l|}
\hline Distance d & $109 \mathrm{~cm}$ & $109 \mathrm{~cm}\left(^{*}\right)$ & $170 \mathrm{~cm}$ & $240 \mathrm{~cm}$ \\
\hline Measurements & $1.3110^{-7}$ & $1.3910^{-7}$ & $5.2610^{-8}$ & $2.1910^{-8}$ \\
\hline MCNPX simulations & $1.1410^{-7}$ & $1.2410^{-7}$ & $4.8010^{-8}$ & $2.4710^{-8}$ \\
\hline
\end{tabular}

$\left(^{*}\right)$ measurement performed with the $500 \mu \mathrm{m}$ thick Si instead of the $4 \mathrm{~mm}$ thick as last detector

Table 1: Measured and simulated yield of protons per source neutron. Typical uncertainties are $+/-6 \%$ for the measurements.

\subsection{Measurements performed as a function of the converter thickness}

In order to optimize the production of recoiling protons, measurements with various thicknesses of the neutron converter were performed. Indeed, protons created at the beginning of a thick converter would be stopped inside it, while a too thin converter would result in a lower yield of protons. $\mathrm{CH}_{2}$ converters of different thickness were tested. Results of measurements and simulations are displayed in Fig. 4. Experimental results are shown as open squares. The dashed line represents the results of simulations with no energy threshold associated with the $\mathrm{Si} 3$ detector. When a $1.5 \mathrm{MeV}$ threshold is applied, the total number of coincidences is lowered by less than $10 \%$. Both experiment and simulation show that the value of the converter thickness should be larger than $1 \mathrm{~mm}$ to optimize the yield of recoil protons. 


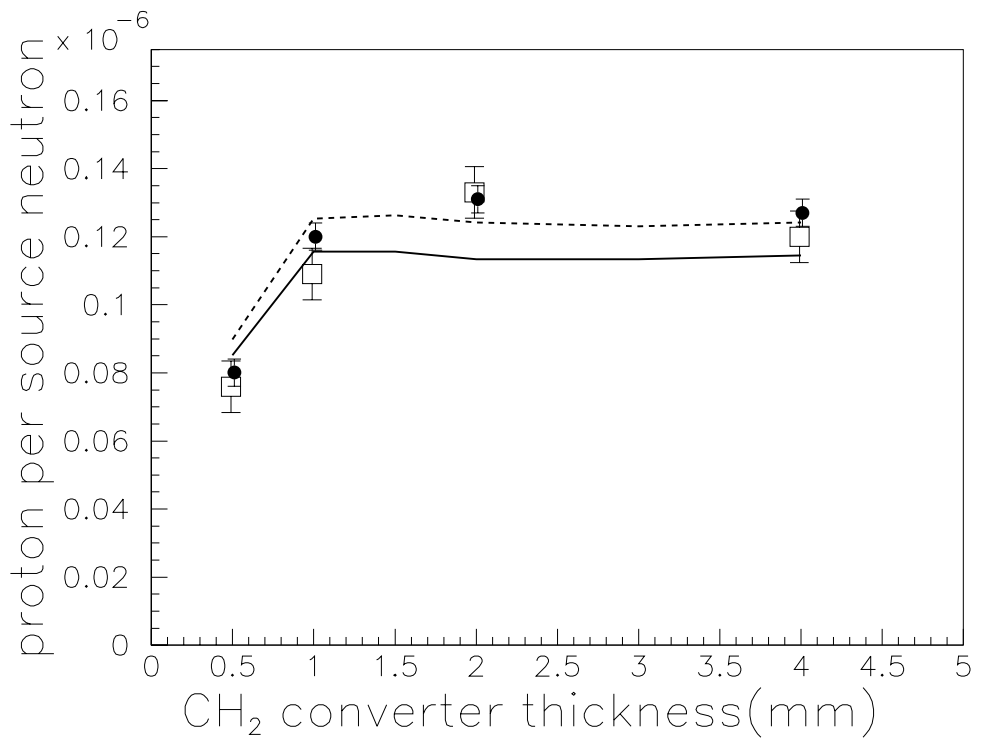

Figure 4: Proton to neutron conversion ratios deduced from the measurements (open squares), from the simulations (dashed line) and from the simulations with a $1.5 \mathrm{MeV}$ energy threshold associated to silicon 3 detector (solid line). The conversion ratios deduced from the scaler values read during the experiment are also plotted (filled circles).

\section{Conclusion}

In this contribution it has been shown that a three element silicon telescope can be valuably used for the monitoring of $14 \mathrm{MeV}$ neutrons. The requirement of a triple coincidence selects unambiguously protons created in the interaction of a neutron beam with a hydrogenous material. Measurements are satisfactorily reproduced by preliminary simulations. Such a device would allow for an on-line relative monitoring of the neutron beam by looking at the number of coincident events per unit of time.

\section{References}

[1] YALINA facility, JIPNR, Sosny, Belarus.

[2] Plateforme d'Etude et de Recherche sur l'Energie Nucléaire, LPSC, Grenoble, France.

[3] $5^{\text {th }}$ EURATOM FP-Contract \#FIKW-CT-2000-00063, Deliverables \# 4 \& 5: MUSE experiments: experimental data set description and physical experimental data set, 2003.

[4] D.B. Pelowitz, MCNPX User's Manual, Version 2.5.0, April 2005, LA-CP-05-0369. 